\title{
Preventative therapies and periodontal interventions for Down syndrome patients
}

\author{
Abstracted from \\ Ferreira R, Michel RC, Greghi SL, Resende ML, Sant'Ana AC, Damante CA, Zangrando MS. \\ Prevention and Periodontal Treatment in Down syndrome patients: A Systematic Review. \\ PLoS One 2016; 11: e0158339. doi:10.1371/journal. pone.0158339 \\ Address for correspondence: Mariana S Zangrando, Division of Periodontics, Department of Prosthodontics, \\ Bauru School of Dentistry, University of São Paulo, Bauru, SP, Brazil. E-mail: mariana@fob.usp.br
}

\section{Question: What type of preventive periodontal programmes provide the best outcomes in Down syndrome patients?}

Data sources Medline, Embase, Cochrane CENTRAL and OpenGREY databases without language restriction until March 2016 plus manual searching of four specific journals and consideration of reference lists. Study selection Studies evaluating different methods of periodontal treatment in Down syndrome patients measuring at least two periodontal parameters at different periods of assessment. Titles, abstracts and full texts were considered by two independent reviewers and a third where discussion did not reach consensus. Randomised controlled trials were evaluated using the Cochrane risk of bias tool. The observational studies were evaluated using an adapted version of the Newcastle-Ottawa Scale.

Data extraction and synthesis Data extraction was carried out independently by two reviewers and organised into evidence tables. No meta-analysis was undertaken, however a narrative synthesis was presented.

Results Nine studies met the inclusion criteria; four longitudinal studies, one prospective case series and four clinical trials which included two cross-over studies and a controlled trial. The studies showed marked heterogeneity in terms of methodology, intervention and outcome measures. All studies, however, included assessment of different plaque and gingival indices.

Three studies investigated outcomes after scaling and root planing, one of which compared surgical and non-surgical approaches. Periodontal pockets of $1-3 \mathrm{~mm}$ were statistically significantly improved with non-surgical treatment in comparison with pockets greater than $4 \mathrm{~mm}$ which showed greater reduction with surgical treatment. Six studies investigated different forms and uses of chlorhexidine, three of which investigated its use as an adjuvant to mechanical debridement and one which also included plaque disclosing as an intervention. Chlorhexidine was shown to be most effective when used daily as a $1 \%$ gel for toothbrushing. The use of a plaque disclosing tablet and fluoridated tooth paste, however, showed further improved outcomes with regards to plaque control.

Conclusions Eight of the nine studies included showed improvement in the primary outcomes of improved plaque and gingival bleeding indices. Professional intervention and periodontal maintenance significantly reduced plaque and gingival indices, irrespective of the treatment performed. Increased frequency of interventions was associated with better outcomes, especially in younger age groups.

\section{Commentary}

Down syndrome (DS) is associated with a range of congenital malformations, altered patterns of growth, variable intellectual disability as well as increased risk of certain chronic diseases. Periodontal disease is one such disease which presents early and severely for those with DS. It has been established that even when matched with individuals of similar intellectual impairment and levels of oral hygiene the DS population will experience more severe periodontitis. ${ }^{1}$ Advances in healthcare have led to dramatic increases in the life expectancy of individuals with DS. This means that tooth loss from chronic periodontal disease, exacerbated by the further deterioration in self-care seen in the likely incidence of the onset of dementia, will be a common challenge for dentists caring for DS patients. This paper reviews the evidence for preventative strategies and periodontal treatment regimes for the DS population.

The authors present an appropriate and logical methodology for the literature search. The detailed narrative and tabular illustration of the PICO scheme and search terms used allows for reproducibility in the future. The two reviewers showed excellent consistency (Kappa $=1$ ) for the nine papers included in this review and make clear the risk of bias associated with each of these studies. The previously adapted Newcastle-Ottawa scale showed that of the five observational studies, none was classed as high quality. When considering the four trials, the Cochrane collaboration tool indicated all were either of high or unclear risk of bias. For each study this information was available at a glance, included in tables also detailing the methods, sample size, interventions and principle results.

Selection bias is unavoidable in the studies, which require good patient compliance with both interventions and measurement indices and also motivated caregivers at home.

The authors were quick to confirm high heterogeneity and low sample sizes and rightfully did not proceed with any pooled analysis of the 279 participants of the various trials. What followed was a protracted narrative synthesis of the included papers.

Given the wide range of periodontal therapies, preventative regimes and potential outcomes affected, it would have seemed logical to identify a more specific intervention or outcome to investigate. However, what was found to be a very limited evidence base may have led the authors to amalgamate the topics. Unfortunately this results in the conclusions of the literature review being lost in this discourse, so we have highlighted these below as practice points. 
Interventions of benefit included supervised tooth-brushing, chlorhexidine gel and using disclosing tablets. These are all low cost interventions with negligible risk to the patient. Whilst methodological insufficiencies may limit reproducibility of the results, nothing is likely to limit the applicability more than the individuality and variability of personal circumstances of those with DS. Practitioners will likely find that interventions need to be tailored to the individual's level of compliance, learning impairment and support at home.

The finding that pockets of greater than $4 \mathrm{~mm}$ respond better with surgical management is noteworthy, but as the potential benefits of interventions must be weighed against associated risks and costs, the fact that this intervention would likely require treatment under general anaesthetic and supported after-care for patients with DS, the applicability in this population is reduced. The review failed to highlight differences in findings both between patients with different levels of intellectual impairment and between patients living in residential care and those living at home. As standards of oral hygiene, and therefore periodontal outcomes, rely so heavily on regular at-home and professional interventions, consideration should really be given to the patients' ability to comply with mouth care and the level of support they have at home.

In general quality of life outcomes were absent from the discussion. Although improvement in plaque and gingival bleeding indices will likely represent reduced active periodontal disease, of greater interest would be the improvement in longer term patient outcomes such as tooth mobility, tooth loss, need for prostheses and discomfort on eating.

The overarching conclusion of this methodologically sound review is the paucity of robust data related to the dental care of those with Down Syndrome. This reflects the dearth of evidencebased dental practice for all groups with physical and learning impairments. It is disappointing that the DS population can suffer a well documented and easily measured disease and yet evidence is still lacking for even basic interventions. Advances in genomics suggest immunological factors play a significant role in the pathogenesis of periodontitis in DS. ${ }^{2}$ It is clear that robust data on well-recognised interventions are needed now whilst we await a clearer immunologically based understanding of how periodontal disease can be prevented for those with DS.

\section{Practice points}

- Preventive oral hygiene programmes and participation of parents/caregivers is key in improving periodontal indices, especially in younger age groups.

- Chlorhexidine is effective used as a mouthrinse or as a $1 \%$ gel used daily, but plaque disclosing tablets present superior outcomes in plaque reduction.

- Increased frequency of professional contact improves periodontal markers.

David Jones ${ }^{a}$ and Joanna Morrison ${ }^{b}$

aPublic Dental Service, NHS Highland, Inverness, Scotland

${ }^{b}$ Public Dental Service, NHS Tayside, Dundee, Scotland

1. Khocht A, Janal M, Turner B. Periodontal health in Down syndrome: contributions of mental disability, personal, and professional dental care. Spec Care Dentist 2010; 30: 118-123.

2. Khocht A. Albandar JM. Aggressive forms of periodontitis secondary to systemic disorders. Periodontol 2000 2014; 65: 134-148.

Evidence-Based Dentistry (2016) 17, 101-102. doi:10.1038/sj.ebd.6401198 